\title{
Singularity of dynamic stress fields around an interface crack between viscoelastic bodies
}

\author{
P.J. WEI ${ }^{1,2}$ and S.Y. ZHANG ${ }^{1}$ \\ ${ }^{1}$ LNM (State Key Laboratory of Nonlinear Mechanics), Institute of Mechanics, Chinese Academy of Sciences, \\ Beijing, P.R. China \\ ${ }^{2}$ Department of Mathematics \& Mechanics, University of Science \& Technology Beijing, P.R. China (E-mail: \\ weipj@126.com)
}

Received 3 May 2002; accepted in revised form 28 January 2004

\begin{abstract}
The singular nature of the dynamic stress fields around an interface crack located between two dissimilar isotropic linearly viscoelastic bodies is studied. A harmonic load is imposed on the surfaces of the interface crack. The dynamic stress fields around the crack are obtained by solving a set of simultaneous singular integral equations in terms of the normal and tangent crack dislocation densities. The singularity of the dynamic stress fields near the crack tips is embodied in the fundamental solutions of the singular integral equations. The investigation of the fundamental solutions indicates that the singularity and oscillation indices of the stress fields are both dependent upon the material constants and the frequency of the harmonic load. This observation is different from the well-known $-1 / 2$ oscillating singularity for elastic bi-materials. The explanation for the differences between viscoelastic and elastic bi-materials can be given by the additional viscosity mismatch in the case of viscoelastic bi-materials. As an example, the standard linear solid model of a viscoelastic material is used. The effects of the frequency and the material constants (short-term modulus, long-term modulus and relaxation time) on the singularity and the oscillation indices are studied numerically.
\end{abstract}

Key words: interface crack, singular integral equation, singularity index, viscoelastic bi-materials

\section{Introduction}

The oscillating singular nature of the stress fields around the crack tips of an interface crack which is located between dissimilar elastic bi-materials and loaded by a static load has been investigated extensively by Williams (1959), Rice and Sih (1965), Erdogan (1965), England (1965), etc. It was found that the singular behavior of the stress fields remains proportional to the inverse square root of $r$ (the radial distance from the crack tip) as in the case of homogeneous materials, but the stress fields possess an oscillatory character, i.e. $\sigma \sim r^{\lambda}=r^{-\frac{1}{2} \pm i \varepsilon}$, where $\varepsilon$ is a function of the material constants. If the materials on both sides of the interface are identical, then $\varepsilon=0$, which means the characteristic parameter $\lambda$ becomes real and the oscillatory nature disappears. The characteristic parameter $\lambda$ of complex value, with the real part (called the singularity index) indicating the singular nature and the imaginary part (called the oscillation index) indicating the oscillatory nature of the stress fields, is completely due to the mismatch of properties of the materials across the interface of bi-materials. The oscillating singularity of stresses around the crack tip applies to the case loaded by a dynamic load as well; the stress fields around the crack tip and the crack opening displacements due to a sud-

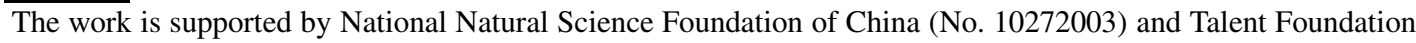
of University of Science \& Technology Beijing. 
denly applied load or harmonic load have been studied by Freund (1974), Srivastava (1978), Kuo (1984) and Qu (1994). However, no attempt has been made to investigate the singular nature of stresses at the crack tips of the interfacial crack between dissimilar viscoelastic bimaterials to our knowledge. Viscoelasticity is a natural framework for modeling dissipative media. The dependence of material responses on the history of loading is generally described by a Boltzmann integral with fading memory. Specially, in the case of time-harmonic load, the constitutive equations of viscoelastic materials reduce to a form which is similar to that of an elastic material, only with the real-valued moduli of an elastic material replaced by the complex-value moduli of a viscoelastic material. The complex moduli of the viscoelastic material are frequency-dependent and thus make the material responses frequency-dependent. In addition, the complex moduli lead to the phase shift between the load and the response. It is the viscosity of material that results in the different mechanical behavior between elastic and the viscoelastic materials. In the viscoelastic interfacial crack problem, the additional viscosity mismatch of materials on both sides of the interface should be taken into account

In the present paper, the influence of the mismatch of viscosity on the singular nature of dynamic stress fields at crack tips of an interfacial crack between dissimilar isotropic viscoelastic bodies loaded by a harmonic load is studied. The outline of the paper is as follows: In Section 2, the mixed boundary valued problem considered is stated. The boundary conditions of displacements and tractions across the interface of bi-materials are prescribed. In Section 3, a set of simultaneous singular integral equations is deduced to model the mixed boundary value problem. The dynamic stress fields around crack tips are studied by an asymptotic analysis and the contact zone size near crack tips is estimated. In Section 4, the standard linear solid model of a viscoelastic material is studied numerically to show the effects of the viscoelastic material constants and the frequency of loading on the singularity and oscillation indices. Finally, some conclusions of interest are summarized in Section 5.

\section{Statement of problem}

Consider a crack of length $2 a$ located at the interface between two dissimilar homogenous, isotropic and linearly viscoelastic bodies, as shown in Figure 1. A Cartesian coordinate system is chosen in such a way that the $x$-axis is along the interface and the origin is located at the middle of the crack. The region, $y>0$, is occupied by a viscoelastic material with the isochoric and dilatational complex moduli, $G_{1}^{c}$ and $K_{1}^{c}$, and density $\rho_{1}$. The region, $y<0$, is occupied by another viscoelastic material with the isochoric and dilatational complex moduli, $G_{2}^{c}$ and $K_{2}^{c}$, and density $\rho_{2}$. It is assumed that the deformation of the composite solids is due to the application of prescribed tractions on the upper and lower surfaces of the interfacial crack, i.e. $|x| \leqslant a$ and $y=0$. The prescribed tractions are assumed harmonic, i.e. $\sigma_{y y}=p(x) e^{i \omega t}$ and $\sigma_{x y}=0$, where $\omega$ is the frequency of loading, $e^{+i \omega t}$ is a time harmonic factor and $p(x)$ indicates the distribution of the normal traction along crack surfaces. It is noted that the normal traction on the crack surfaces is pressure and tension alternately and thus makes the crack open and close alternately. In order to avoid any complicated contact boundary conditions, it is assumed that the crack is open in advance and remains open while the harmonic load is imposed. Then, the boundary conditions of displacements and tractions on the interface can be expressed as

$$
\begin{aligned}
& \boldsymbol{\sigma}\left(x, 0^{+}, \omega\right)=\boldsymbol{\sigma}\left(x, 0^{-}, \omega\right)=\left(p(x) e^{i \omega t}, 0\right) \quad|x| \leq a \\
& \sigma\left(x, 0^{+}, \omega\right)=\sigma\left(x, 0^{-}, \omega\right) \quad|x|>a
\end{aligned}
$$




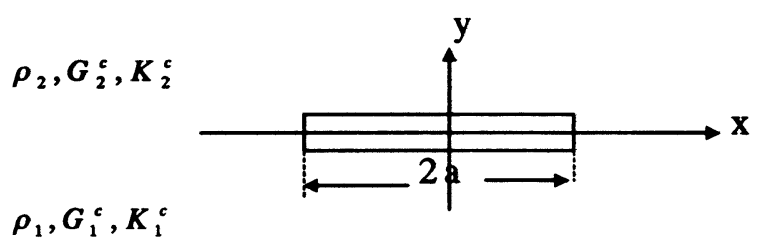

Figure 1. An interface crack between two dissimilar viscoelastic bodies.

$$
\begin{array}{ll}
\mathbf{u}\left(x, 0^{+}, \omega\right)-\mathbf{u}\left(x, 0^{-}, \omega\right) & |x|>a \\
\mathbf{u}(x, y, \omega)=0 & x, y \rightarrow \infty,
\end{array}
$$

where $\sigma=\left(\sigma_{y y}, \sigma_{x y}\right)$ and $\mathbf{u}=\left(u_{y}, u_{x}\right)$ are the traction and displacement vectors.

In the case of harmonic loading, both of the two-dimensional displacement fields and stress fields in the viscoelastic solids are also harmonic with the same time harmonic factor $e^{i \omega t}$. The equation of motion in viscoelastic solids is

$$
\left(K^{c}(\omega)+\frac{1}{3} G^{c}(\omega)\right) \nabla(\nabla \cdot \mathbf{u}(x, y, \omega))+G^{c}(\omega) \nabla^{2} \mathbf{u}(x, y, \omega)+\rho \omega^{2} \mathbf{u}(x, y, \omega)=0,
$$

where $\nabla$ and $\nabla^{2}$ are the gradient operator and the Laplacian operator, respectively. $G^{c}(\omega)$ and $K^{c}(\omega)$ are the frequency-dependent isochoric and dilatational complex moduli of the viscoelastic material. The viscosity of material is embodied in the imaginary part of the complex moduli and results in the phase shift between the complex stresses and the complex strains in viscoelastic solids. This can be seen clearly from the constitutive relations of a viscoelastic material

$$
\begin{aligned}
& s_{i j}^{c}(x, y, \omega)=2 G^{c}(\omega) e_{i j}^{c}(x, y, \omega) \\
& \sigma_{k k}^{c}(x, y, \omega)=3 K^{c}(\omega) \varepsilon_{k k}^{c}(x, y, \omega),
\end{aligned}
$$

where $\varepsilon_{i j}^{c}, \sigma_{i j}^{c}, e_{i j}^{c}, s_{i j}^{c}$ are strain, stress, deviatoric strain and deviatoric stress in complex form, respectively. Substituting the Helmholtz representation of the displacement vector $\boldsymbol{u}$

$$
\boldsymbol{u}(x, y, \omega)=\nabla \Phi(x, y, \omega)+\nabla \times \boldsymbol{\Psi}(x, y, \omega) \quad(\nabla \cdot \boldsymbol{\Psi}=0)
$$

into Eq. (2) yields the dilatational and isochoric wave equations:

$$
\begin{aligned}
& \nabla^{2} \varphi(x, y, \omega)+k_{p}^{2} \varphi(x, y, \omega)=0 \\
& \nabla^{2} \boldsymbol{\Psi}(x, y, \omega)+k_{s}^{2} \boldsymbol{\Psi}(x, y, \omega)=0,
\end{aligned}
$$

where $k_{p}=\omega / c_{p}$ and $k_{s}=\omega / c_{s}$ are the complex-valued wavenumbers of the dilatational $(p)$ and isochoric $(s)$ waves, respectively. $c_{p}=p \cdot v \cdot\left[K^{c}(\omega)+\frac{4}{3} G^{c}(\omega)\right]^{\frac{1}{2}} / \rho^{\frac{1}{2}}$ and $c_{s}=$ $p \cdot v \cdot\left[G^{c}(\omega)\right]^{\frac{1}{2}} / \rho^{\frac{1}{2}}$ are the complex-valued wave velocities of the $p$ and $s$ wave, respectively. $\rho$ is the density of the material. $p \cdot v[]$ denotes the principle value. $\varphi$ is the scalar potential of the dilatational wave and $\boldsymbol{\Psi}$ is the vector potential of the isochoric wave. 


\section{The dynamic stress fields and the contact zone near crack-tips}

\subsection{THE DEDUCTION OF SINGULAR INTEGRAL EQUATIONS}

For convenience, the common time harmonic factor $e^{i \omega t}$ is omitted in the following, but understood. After the Fourier transformation is performed on $x$ in Eq. (5) and the radiation condition (1d) is used, the potential functions in the transformed domain are obtained as

$$
\begin{aligned}
& \bar{\varphi}(s, y)= \begin{cases}A_{1}(s) e^{-\alpha_{1} y} & y>0 \\
A_{2}(s) e^{\alpha_{2} y} & y<0\end{cases} \\
& \bar{\psi}(s, y)= \begin{cases}B_{1}(s) \mathbf{z e}^{-\beta_{1} y} & y>0 \\
B_{2}(s) \mathbf{z e}^{\beta_{2} y} & y<0,\end{cases}
\end{aligned}
$$

where $\alpha_{l}=\sqrt{s^{2}-k_{p l}^{2}}$ and $\beta_{l}=\sqrt{s^{2}-k_{s l}^{2}}$. The subscript $l$ indicates the specific semi-infinite regions, i.e. $l=1$ for $y>0$ and $l=2$ for $y<0 . \bar{f}(s)=F(f(x))$ indicates the Fourier transformation of a physical quantity $f$ with respect to $x$ and $s$ is the transform parameter. $\mathbf{z}$ is the unit vector of the $z$-axis. $A_{l}(s)$ and $B_{l}(s)$ are the functions of $s$ and will be determined from the boundary conditions. Recalling Eq. (4), the displacements in the transformed domain can be expressed as

$$
\left\{\begin{array}{c}
\bar{u}_{x l} \\
\bar{u}_{y l}
\end{array}\right\}=\left[R_{l}\right]\left\{\begin{array}{c}
A_{l} \\
B_{l}
\end{array}\right\}=\left[C_{l}\right] \cdot\left[E_{l}\right]\left\{\begin{array}{c}
A_{l} \\
B_{l}
\end{array}\right\}(l=1,2),
$$

where

$$
\left[C_{l}\right]=\left[\begin{array}{cc}
i s & (-1)^{l} \beta_{l} \\
(-1)^{l} \alpha_{l} & (-1)^{l}(i s)
\end{array}\right],\left[E_{l}\right]=\left[\begin{array}{ll}
e^{(-1)^{l} \alpha_{l} y} & 0 \\
0 & e^{(-1)^{l} \beta_{l} y}
\end{array}\right] .
$$

By employing the constitutive relations (3), the stresses in the transformed domain can be expressed as

$$
\left\{\begin{array}{c}
\bar{\sigma}_{y y l} \\
\bar{\sigma}_{x y l}
\end{array}\right\}=\left[T_{l}\right]\left\{\begin{array}{c}
A_{l} \\
B_{l}
\end{array}\right\}=\left[D_{l}\right] \cdot\left[E_{l}\right]\left\{\begin{array}{c}
A_{l} \\
B_{l}
\end{array}\right\}(l=1,2),
$$

where

$$
\left[D_{l}\right]=\left[\begin{array}{lc}
\left(K_{l}^{c}(\omega)-\frac{2}{3} G_{l}^{c}(\omega)\right)\left(\alpha_{l}^{2}-s^{2}\right)+2 G_{l}^{c}(\omega) \alpha_{l}^{2} & i 2 s(-1)^{l} G_{l}^{c}(\omega) \beta_{l} \\
2 s(-1)^{l} G_{l}^{c}(\omega) \alpha_{l} & \left(\beta_{l}^{2}+s^{2}\right) G_{l}^{c}(\omega)
\end{array}\right] .
$$

The tractions on the interface are obtained from Eq. (8) by letting $y=0$, i.e.

$$
\{\bar{Q}\}=\left[D_{1}\right]\left\{\begin{array}{l}
A_{1} \\
B_{1}
\end{array}\right\}=\left[D_{2}\right]\left\{\begin{array}{l}
A_{2} \\
B_{2}
\end{array}\right\}
$$

The crack displacement jump vector is defined as

$$
\Delta \mathbf{u}(x)=\mathbf{u}\left(x, 0^{+}\right)-\mathbf{u}\left(x, 0^{-}\right)
$$


in the original domain, and can be expressed as

$$
\Delta \overline{\boldsymbol{u}}=\left(\left[C_{1}\right] \cdot\left[D_{1}\right]^{-1}-\left[C_{2}\right] \cdot\left[D_{2}\right]^{-1}\right)\{\bar{Q}\}=[M]^{-1}\{\bar{Q}\}
$$

in the transformed domain by the use of Eq. (7) and Eq. (9); Eq. (11) relates the crack displacement jumps to the tractions on the surfaces of the crack. The boundary conditions of the displacement and the stress, (1c) and (1a), construct a set of dual integral equations for the crack displacement jumps.

$$
\begin{array}{ll}
F^{-1}(\Delta \overline{\mathbf{u}})=\mathbf{0} & |x|>a \\
F^{-1}([M] \Delta \overline{\mathbf{u}})=\sigma_{0}(x) & |x| \leq a,
\end{array}
$$

where $F^{-1}()$ indicates the inverse Fourier transformation. $\sigma_{0}(x)=\left(p(x) e^{i w t}, 0\right)$ is the prescribed traction.

In order to obtain a set of singular integral equations from Eq. (12), the crack dislocation density vector defined in the original domain

$$
\mathbf{U}(x)=\frac{\partial}{\partial x} \boldsymbol{\Delta} \boldsymbol{u}(\boldsymbol{x})
$$

is introduced. Performing the Fourier transformation on Eq. (13) and then inserting it into Eq. (12) leads to equations

$$
\begin{array}{ll}
F^{-1}\left(\frac{1}{i s} \overline{\mathbf{U}}\right)=\mathbf{0}, & |x|>a \\
F^{-1}\left(\frac{1}{i s}[M] \overline{\mathbf{U}}\right)=\sigma_{0}(x) & |x| \leq a
\end{array}
$$

The following function limit when $s \rightarrow \pm \infty$ is provided by $\mathrm{Qu}$ (1994)

$$
\lim _{s \rightarrow \pm \infty} \frac{1}{s}[M]=\left[\begin{array}{cc}
-\operatorname{sgn}(s) m & i m \beta \\
-i m \beta & -\operatorname{sgn}(s) m
\end{array}\right]=m\left(i \beta\left[I_{0}\right]-\operatorname{sgn}(s)[I]\right),
$$

where

$$
\begin{aligned}
& {\left[I_{0}\right]=\left[\begin{array}{cc}
0 & 1 \\
-1 & 0
\end{array}\right],[I]=\left[\begin{array}{ll}
1 & 0 \\
0 & 1
\end{array}\right], m=G_{1}^{c}(\omega)\left(\frac{1}{\left(1-r_{1}^{2}\right)}\left(\frac{1+\alpha}{1-\beta^{2}}\right),\right.} \\
& \alpha=\frac{G_{2}^{c}(\omega) r_{1}^{2}\left(r_{2}^{2}-1\right)-G_{1}^{c}(\omega) r_{2}^{2}\left(r_{1}^{2}+1\right)}{G_{2}^{c}(\omega) r_{1}^{2}\left(r_{2}^{2}-1\right)+G_{1}^{c}(\omega) r_{2}^{2}\left(r_{1}^{2}+1\right)}, \beta=\frac{G_{2}^{c}(\omega)\left(r_{2}^{2}-1\right)-G_{1}^{c}(\omega)\left(r_{1}^{2}-1\right)}{G_{2}^{c}(\omega) r_{1}^{2}\left(r_{2}^{2}-1\right)+G_{1}^{c}(\omega) r_{2}^{2}\left(r_{1}^{2}-1\right)}, \\
& r_{l}^{2}=\frac{3 K_{l}^{c}(\omega)+4 G_{l}^{c}(\omega)}{3 G_{l}^{c}(\omega)}(l=1,2)
\end{aligned}
$$

By employing Eq. (15) and the following equations (Qu, 1994)

$$
\int_{-\infty}^{\infty} \exp (i \xi x) \mathrm{d} \xi=2 \pi \delta(x)
$$




$$
\int_{-\infty}^{\infty} \operatorname{sgn}(\xi) \exp (i \xi x) \mathrm{d} \xi=\frac{2 i}{x}
$$

a set of simultaneous singular integral equations in terms of crack dislocation densities, $\mathbf{U}=$ $\left(U_{y}, U_{x}\right)$, are obtained from Eq. (14b)

$$
\beta\left[I_{0}\right] \mathbf{U}-\frac{1}{\pi} \int_{-a}^{a} \frac{1}{\xi-x} \mathbf{U d} \xi+\int_{-a}^{a}[K(\xi, x)] \mathbf{U d} \xi=\frac{1}{m} \sigma_{0}(x) \quad|x|<a,
$$

where

$$
[K(\xi, x)]=-\frac{i}{2 \pi} \int_{-\infty}^{\infty}\left\{\frac{1}{s m}[M]-i \beta\left[I_{0}\right]+\operatorname{sgn}(s)[I]\right\} e^{i s(\xi-x)} \mathrm{d} s .
$$

For the purpose of uncoupling, a new dislocation density, i.e. $\lambda(x)=U_{y}(x)-i U_{x}(x)$, is introduced. Then, two equations in Eq. (17) are incorporated into one.

$m \beta \lambda(x)-\frac{i m}{\pi} \int_{-a}^{a} \frac{\lambda(\xi)}{\xi-x} \mathrm{~d} \xi+\frac{m}{2} \int_{-a}^{a} L_{1}(x, \xi) \lambda(\xi)+L_{2}(x, \xi) \bar{\lambda}(\xi) \mathrm{d} \xi=q(x) \quad|x| \leqslant a$,

where $q(x)=\sigma_{y y}+i \sigma_{x y}=p(x), \quad L_{1}=\left(K_{11}-K_{21}\right)+i\left(K_{11}+K_{22}\right), \quad L_{2}=\left(K_{12}+\right.$ $\left.K_{21}\right)+i\left(K_{22}-K_{11}\right), \quad \bar{\lambda}(x)=U_{y}(x)+i U_{x}(x)$.

Equation (14a) can be represented as $\int_{-a}^{a} U(x) \mathrm{d} x=0$ or, further,

$$
\int_{-a}^{a} \lambda(x) \mathrm{d} x=0
$$

which ensures the unique solution of the singular integral equation.

\subsection{THE STRESS FIELDS NEAR CRACK TIPS}

Equation (18) is obtained from the constitutive relations and the equation of motion for the boundary $|x| \leqslant a$. However, it can be extended to the boundary $|x| \geqslant a$. Considering the displacement continuous conditions, i.e. $\lambda(x)=0$ for $|x| \geqslant a$, and the regularization of crack length, i.e. marking $x / a$ as $x$ still, the counterpart of Eq. (18) for the boundary $|x| \geqslant a$ can be expressed as

$\sigma_{y y}+i \sigma_{x y}=-\frac{i m}{\pi} \int_{-1}^{1} \frac{\lambda(\xi)}{\xi-x} \mathrm{~d} \xi+\frac{m}{2} \int_{-1}^{1} L_{1}(x, \xi) \lambda(\xi)+L_{2}(x, \xi) \bar{\lambda}(\xi) \mathrm{d} \xi \quad|x| \geqslant 1$

It is noted that the right second term of Eq. (20) is finite when $x \rightarrow \pm 1$. The singular nature of stresses near crack tips is embodied in the first term, i.e.

$$
\sigma_{y y}+i \sigma_{x y} \approx-\frac{i m}{\pi} \int_{-1}^{1} \frac{\lambda(\xi)}{\xi-\pi} \mathrm{d} \xi \quad x \rightarrow \pm 1
$$

According to the discussion on the singular integral equation given by Muskhelishvili (1953) and Erdogan et al. (1973), the unknown function $\lambda(x)$ can be expressed in the form of a series:

$$
\lambda(x)=\sum_{n=0}^{\infty} c_{n} W(x) P_{n}^{\left(\eta_{1}, \eta_{2}\right)}(x),
$$


where $P_{n}^{\left(\eta_{1}, \eta_{2}\right)}(x)$ is the Jacobi polynomial, and $c_{n}$ are the unknown expansion coefficients. $W(x)$ is the fundamental solution of the singular integral equation, and can be generally expressed as

$$
W(x)=(1-x)^{\eta_{1}}(1+x)^{\eta_{2}},
$$

where the powers, $\eta_{1,2}=\delta \pm i \varepsilon$, are determined by the coefficients of the singular integral equation, i.e.

$$
\begin{aligned}
& \varepsilon=-\frac{1}{2 \pi} \ln \left|\frac{\beta-1}{\beta+1}\right| \\
& \delta=-\frac{1}{2 \pi} \arg \left(\frac{\beta-1}{\beta+1}\right) \quad(\arg () \text { indicates argument of a complex number })
\end{aligned}
$$

After inserting Eq. (22) into Eq. (21), and employing the following equation (Srivastava, 1978)

$$
\frac{1}{\pi i} \int_{-1}^{1} P_{n}^{\left(\eta_{1}, \eta_{2}\right)}(t) W(t) \frac{\mathrm{d} t}{t-x}=(1-\beta)\left[W(x) P_{n}^{\left(\eta_{1}, \eta_{2}\right)}(x)-G_{n}^{\infty}(x)\right] \quad|x|>1,
$$

where $G_{n}^{\infty}(x)$ is the main part of $W(x) P_{n}^{\left(\eta_{1}, \eta_{2}\right)}(x)$ at infinity, the asymptotic expression of stress fields near the crack tips can be written as

$$
\sigma_{y y}+i \sigma_{x y} \approx m(1-\beta)\left[W(x) \sum_{n=0}^{\infty} c_{n} P_{n}^{\left(\eta_{1}, \eta_{2}\right)}(x)\right] \quad x \rightarrow \pm 1
$$

It can be seen clearly from Eq. (26) that the singular nature of the dynamic stress fields is completely characterized by the fundamental solution $W(x)$. Therefore, we will focus our discussion on the fundamental solution $W(x)$ in the following. Considering that $(1-x)^{i \varepsilon}$ can be rewritten as $e^{i \varepsilon \ln (1-x)}$, we know the stress fields near the crack tips are oscillatory if $\varepsilon$ does not equal zero. It is noted that the parameter $\beta$ equals zero when the materials (elastic or viscoelastic) on both sides of the interface are the same. This makes the oscillatory index $\varepsilon$ equal to zero, and the oscillatory feature of stresses disappears. However, the parameter $\beta$ does not equal zero when the materials on both sides of the interface are different. This results in the well-known oscillatory features of the stress fields near the crack tips. Further, in the case of elastic bi-materials, the parameter $\beta$ is real-valued because both the dilatational and the isochoric moduli are real-valued. However, in the case of viscoelastic bi-materials, the parameter $\beta$ is complex-valued because both the dilatational modulus, $K_{i}(\omega)$, and the isochoric modulus, $G_{i}(\omega)$, are complex functions of the frequency $\omega$. It is known from Eq. (24) that the singularity index $\delta$ is no longer constant $(-1 / 2)$ but dependent on material constants, as is oscillation index $\varepsilon$. Also the singularity and oscillation indices are frequency-dependent due to the fact that the complex moduli of viscoelastic materials are frequency-dependent.

\subsection{THE CONTACT ZONE NEAR THE CRACK TIPS}

It follows from Eqs. (10), (13) and (22) that the crack displacement jumps are given by

$$
\Delta u_{y}(x)-i \Delta u_{x}(x)=\sum_{n=0}^{\infty} c_{n}\left[\int_{-1}^{x} w(\xi) P_{n}^{\left(\eta_{1}, \eta_{2}\right)}(\xi) \mathrm{d} \xi\right] .
$$


The near-tip behavior can be examined by letting $x \rightarrow \pm 1$. For example, at the right crack tip $(x=1)$, the crack displacement jumps can be written asymptotically as $(\mathrm{Qu}, 1994)$

$$
\Delta u_{y}(x)-i \Delta u_{x}(x) \approx-\sqrt{\frac{1-x}{2}}\left(\frac{x-1}{x+1}\right)^{-i \varepsilon} \sum_{n=0}^{\infty} c_{n} P_{n}^{\left(\eta_{1}, \eta_{2}\right)}(1) \quad x \rightarrow 1
$$

Considering

$$
\left(\frac{x-1}{x+1}\right)^{-i \varepsilon}=e^{-i \varepsilon \ln \left(\frac{x-1}{x+1}\right)}
$$

we note that the crack displacement jump changes its sign an infinite number of times when $x$ approaches the crack tip $(x \rightarrow 1)$. This indicates the well-known interpenetration of crack surfaces, which contradicts the assumption that no contact zone is created during the application of the harmonic load. However, if the contact zone is sufficiently small, the present solution can be considered to reach the actual solution sufficiently well. The contact zone size, $|x|<x_{c}<1$, can be estimated by finding $x_{c}$ where the crack opening displacement satisfies $\Delta u_{y}\left(x_{c}\right)=0$. It leads to

$$
R_{e}\left(\frac{x_{c}-1}{x_{c}+1}\right)^{-i \varepsilon}=0
$$

Its solution is obtained immediately

$$
\frac{l_{c}}{L_{c}}=\frac{1-x_{c}}{1+x_{c}}=e^{-\frac{\pi}{2 \varepsilon}},
$$

where $l_{c} / L_{c}$ indicates the ratio of the distances from $x_{c}$ to both of the crack tips. In general, the value of the oscillation index $\varepsilon$ is considerably small for various combinations of practical bi-materials. This has been discussed by Rice (1988). As an example, let $\varepsilon=0.025$, the corresponding contact zone is estimated from Eq. (31) as $l_{c} / L_{c}=5.1582 \times 10^{-28}$. The contact zone size will decrease with the decrease of the oscillation index $\varepsilon$.

\section{A numerical example}

In order to investigate the influence of the viscoelastic material constants and the frequency of loading on the singularity and oscillation indices, a numerical example is given in which the standard linear solid model of a viscoelastic material is used. The isochoric relaxation function and the corresponding complex modulus are

$$
\begin{aligned}
& G(t)=\mu_{\infty}+\left(\mu_{0}-\mu_{\infty}\right) \exp \left(-\frac{t}{\tau}\right) \\
& G^{c}(\omega)=i \omega F(G(t))=\frac{\mu_{\infty}+i \mu_{0} \omega \tau}{1+i \omega \tau},
\end{aligned}
$$

where $\tau$ is the relaxation time. $\mu_{0}$ and $\mu_{\infty}$ are the short-term and the long-term modulus, respectively. For convenience, the Poisson ratio $v$ is assumed constant. Then, the complex dilatational modulus can be expressed as

$$
K^{c}(\omega)=\frac{2(1+v)}{3(1-2 v)} G^{c}(\omega) .
$$


A viscoelastic material with material constants, $\mu_{0}=1060\left(\mathrm{MNm}^{-2}\right), \mu_{\infty}=264\left(\mathrm{MNm}^{-2}\right)$, $\tau=1(\mathrm{~s}), v=0.3$, and density $\rho=1200\left(\mathrm{kgm}^{-3}\right)$, is considered and is assumed to be located on one side of the interface. We take the viscoelastic material on another side of the interface with only one of these material constants, i.e. $\mu_{0}, \mu_{\infty}$ or $\tau$, different and the rest remain the same to form various combinations of bi-materials. Then, the singularity index $\delta$ and the oscillation index $\varepsilon$ are evaluated for these combinations of bi-materials, which is indicated by the ratio of material constants, i.e. $\mu_{10} / \mu_{20}, \mu_{1 \infty} / \mu_{2 \infty}$ and $\tau_{1} / \tau_{2}$. Here, the first subscript $l$ of $\mu_{l s}$ indicates which side of the interface the material is located on, i.e. $l=1$ for $y>0$ and $l=2$ for $y<0$, and the second subscript $s$ distinguishes between the shortterm and the long-term modulus. It is noted that the homogeneous material can be recovered by letting $\mu_{10} / \mu_{20}=1, \mu_{1 \infty} / \mu_{2 \infty}=1$ and $\tau_{1} / \tau_{2}=1$. Also, the elastic bi-materials can be recovered by letting $\tau_{1}=\tau_{2}=\infty$ or $\mu_{l 0}=\mu_{l \infty}(l=1,2)$. In addition, the viscosity of the materials (characterized by the imaginary part of the complex modulus) is pronounced only within a finite range of frequency for the standard solid model, as shown in Figure 2a, Figure $3 \mathrm{a}$ and Figure $4 \mathrm{a}$. We call this range the effective influence range of frequency. Outside the effective influence range of frequency, the viscosity of the material can be ignored and the viscoelastic material is nearly identical to the elastic material. The evaluation of the singularity index and the oscillation index is thus restricted to the effective influence range of frequency in the present numerical example.

The effects of short-term modulus $\mu_{0}$ are shown in Figure 2. Curve 1 in Figure 2a denotes the complex isochoric modulus of the viscoelastic material on one side of the interface. The complex isochoric modulus of the viscoelastic material on another side is denoted by curves $2 \sim 4$, respectively, which correspond to three short-term moduli, i.e. $\mu_{20}=1.1 \mu_{10}$, $1.3 \mu_{10}$ and $1.5 \mu_{10}$, with the long-term modulus and the relaxation time fixed, i.e. $\mu_{2 \infty}=\mu_{1 \infty}$, $\tau_{2}=\tau_{1}$. The singularity index $\delta$ and the oscillation index $\varepsilon$ corresponding to the three cases of material combination are shown in Figure $2 \mathrm{~b}$ and Figure $2 \mathrm{c}$. It is shown that the singularity index $\delta$ tends to $-1 / 2$ at relatively high and low frequencies outside the effective influence range of frequency. This phenomenon can be explained by the fact that the complex modulus tends to the real-valued short-term modulus $\mu_{0}$ at a relatively high frequency and the realvalued long-term modulus $\mu_{\infty}$ at a relatively low frequency, respectively. Within the effective influence range of frequency, the singularity index $\delta$ increases when the modulus difference, $\mu_{20} / \mu_{10}$, increases. Similarly, the oscillation index $\varepsilon$, which is dependent upon the shortterm modulus difference at a relatively high frequency, increases when the modulus difference increases.

The effects of long-term modulus $\mu_{\infty}$ are shown in Figure $3\left(\mu_{21} / \mu_{11}=\mu_{2 \infty} / \mu_{1 \infty}\right)$. For three cases of materials combination, i.e. $\mu_{2 \infty}=1.1 \mu_{1 \infty}, 1.3 \mu_{1 \infty}$ and $1.5 \mu_{1 \infty}$, the singularity index $\delta$ tends to $-1 / 2$ at a relatively high and low frequency outside the effective influence range of frequency, as shown in Figure $3 b$, and can be explained invariably by the fact that viscoelastic material reduces nearly to elastic material outside the effective influence range of frequency. Within the effective influence range of frequency, the singularity index $\delta$ decreases (its absolute value increases) when the long-term modulus difference, $\mu_{2 \infty} / \mu_{1 \infty}$, increases. The oscillation index $\varepsilon$, which is dependent on the long-term modulus difference at a relatively low frequency, increases when the long-term modulus difference increases, as shown in Figure 3c.

The effects of relaxation time $\tau$ are shown in Figure 4. Because the materials on both sides of the interface tend to be identical at a relatively high and low frequency outside the effective influence range of frequency, the singularity index $\delta$ tends to $-1 / 2$, as shown in Figure $4 \mathrm{~b}$, 


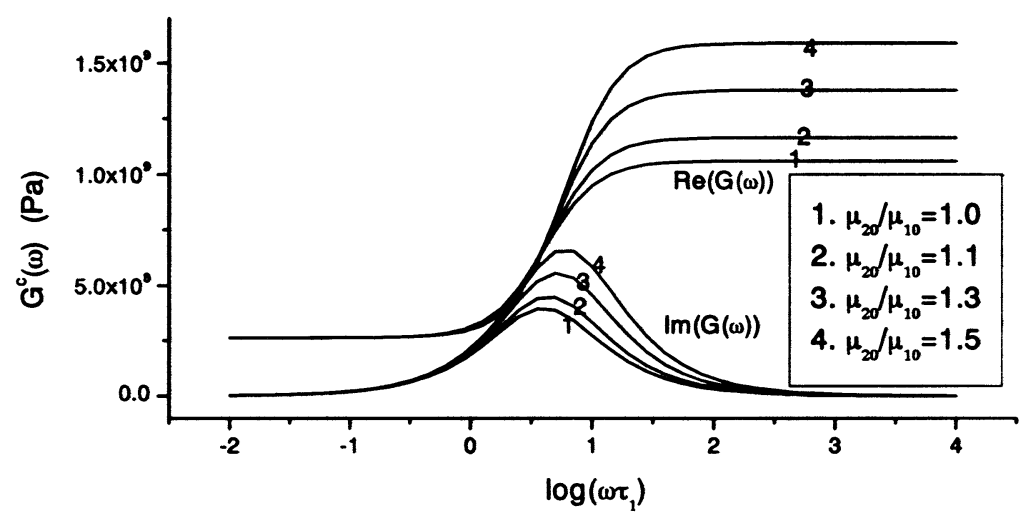

(a)

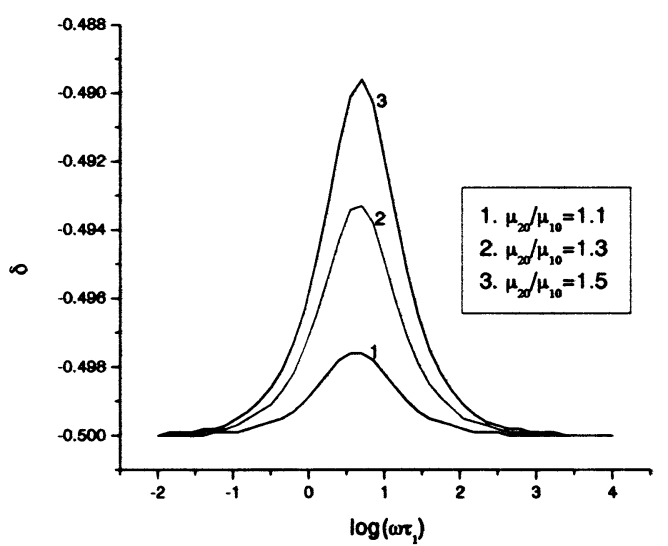

(b)

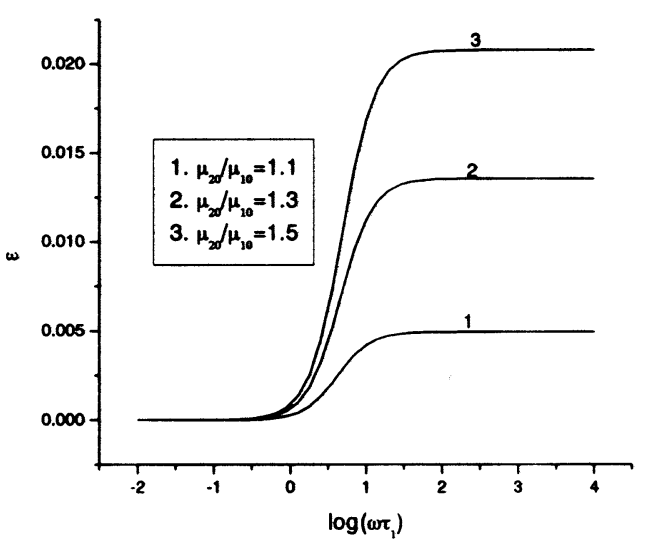

(c)

Figure 2. The effects of short-term modulus $\mu_{0}$. a) The effects on complex modulus $G^{c}(\omega)$. b) The effects on singular index $\delta$.c) The effects on oscillatory index $\varepsilon$.

and the oscillation index $\varepsilon$ tends to zero, as shown in Figure 4c. Within the effective influence range of frequency, the oscillation index $\varepsilon$ increases with the increase of the relaxation time difference, i.e. $\tau_{2} / \tau_{1}$. This indicates that the magnitude of oscillation index $\varepsilon$ characterizes the difference of the mechanical properties of bi-materials. A phenomenon of interest, which can be seen clearly from Figure $4 \mathrm{~b}$, is that the singularity index $\delta$ waves around $-1 / 2$ within the effective influence range of frequency. This is considered a consequence of the mismatch of viscosity of the materials on both sides of the interface. We noted that the viscosity of materials on both sides of the interface changes with frequency, respectively. At a relatively low frequency, the viscosity of material on one side of the interface is greater than that on another side. However, the situation inverses at a relatively high frequency. The turning points, where the viscosities of materials on both sides of the interface are identical, correspond to different frequencies for the three cases of materials combination. Therefore, we think with reason that the phenomenon that singularity index $\delta$ waves around $-1 / 2$ within the effective influence range of frequency, is due to the change of the mismatch pattern of viscosity of the materials on both sides of the interface. This is a salient feature of viscoelastic bi-materials 


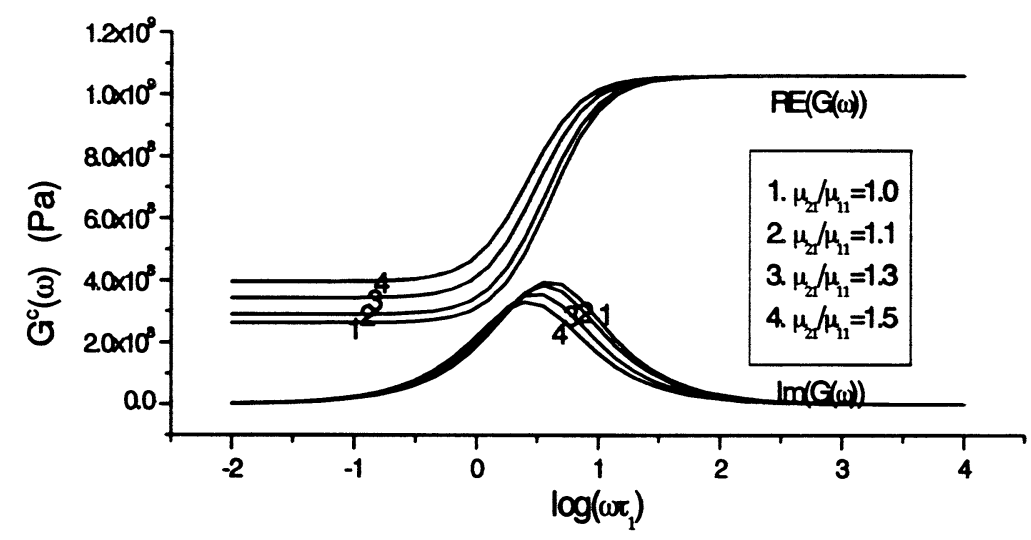

(a)

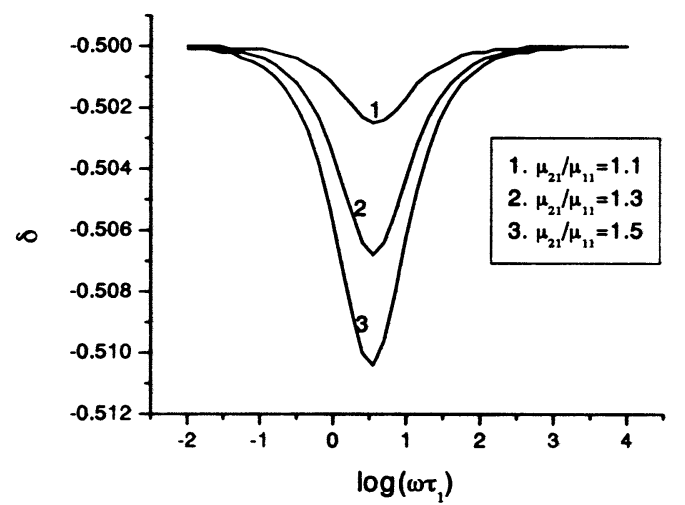

(b)

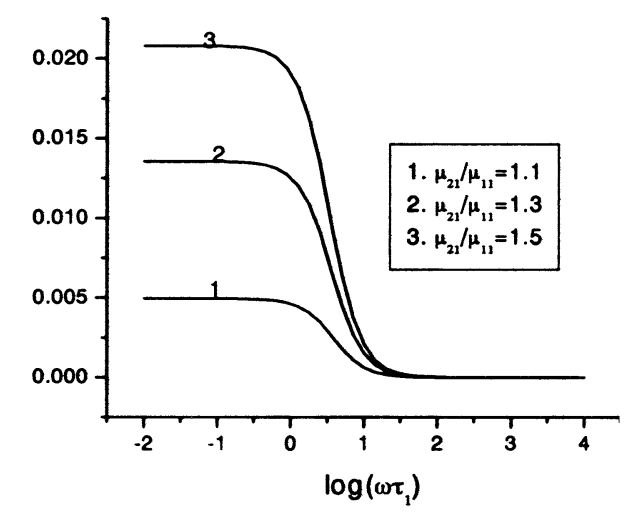

(c)

Figure 3. The effects of long-term modulus $\mu_{\infty}$. a) The effects on complex modulus $G^{c}(\omega)$. b) The effects on singular index $\delta$. c) The effects on oscillatory index $\varepsilon$.

versus elastic bi-materials. In addition, we noted that the singularity index $\delta$ equals $-1 / 2$ at the turning points mentioned above, where the viscosity of materials on both sides of the interface does not disappear but is identical. It implies that the singularity index is only affected by the viscosity mismatch of materials but independent of the amplitude of viscosity of the materials on both sides of the interface.

The oscillation index $\varepsilon$ characterizes the difference of mechanical properties of the materials on both sides of the interface, as shown in the numerical example. But it can also be considered as a characteristic parameter of the size of the contact zone near crack tips from the discussion in Section 3.3. The size of the contact zone will increase with the increase of the oscillation index $\varepsilon$. It is noted that the oscillation index $\varepsilon<0.025$ for various bi-materials combinations which are considered in the present numerical example. This means that the size of the contact zone near the crack tip is not greater than $l_{c} / L_{c}=5.1582 \times 10^{-28}$ (obtained in Section 3.3) for various bi-materials combinations considered. Although no contact boundary is considered in the boundary conditions prescribed, it is considered that the present solution is close enough to the actual solution for such a small contact zone and thus ensures the validity of the present discussion. 


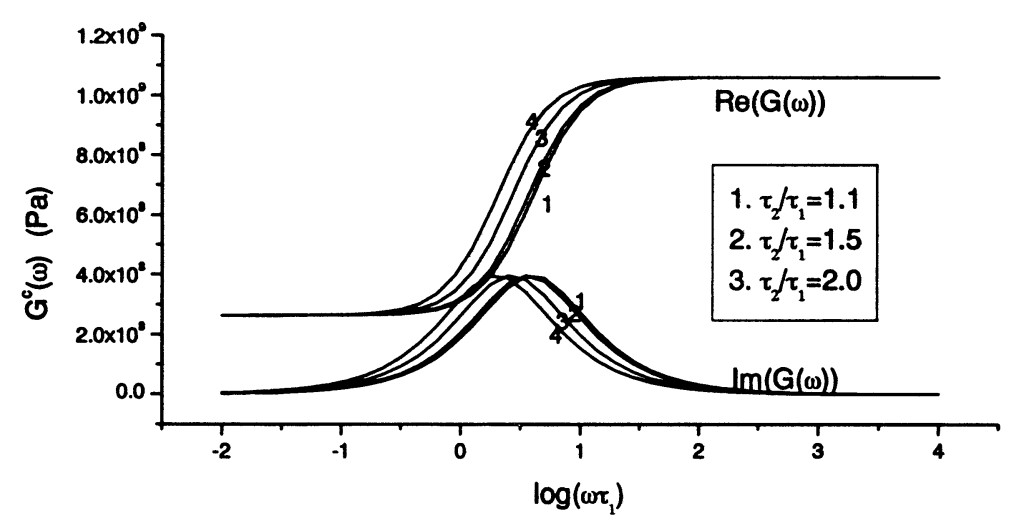

(a)

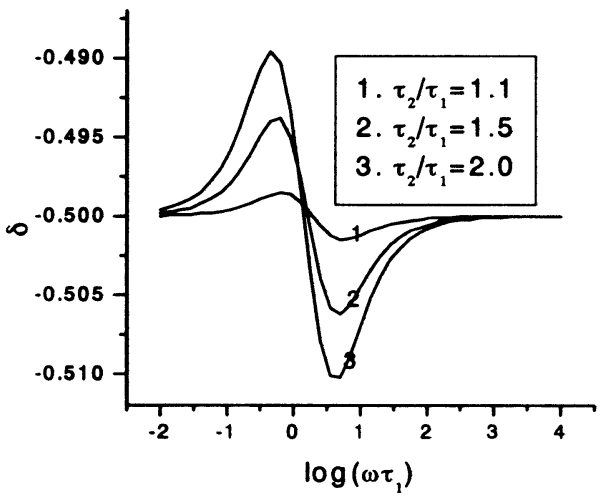

(b)

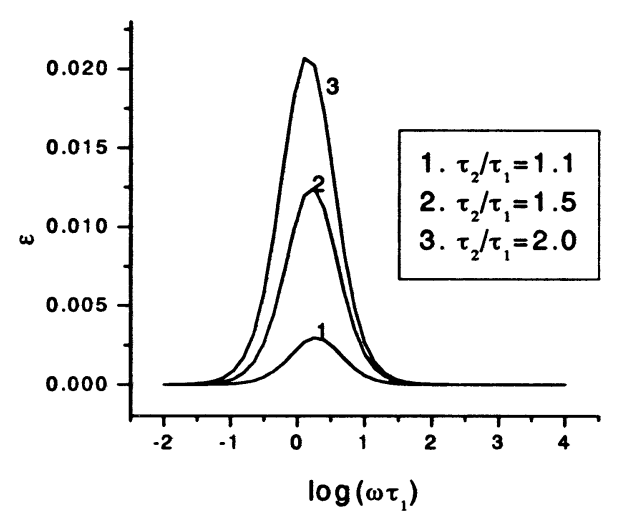

(c)

Figure 4. The effects of relaxation time $\tau$. a) The effects on complex modulus $G^{c}(\omega)$. b) The effects on singular index $\delta$. c) The effects on oscillatory index $\varepsilon$.

\section{Concluding remarks}

It is well known that the stress fields around the crack tip of an interfacial crack between elastic bi-materials are of oscillating singular nature. This is applicable to an interfacial crack between viscoelastic bi-materials too. However, the singularity and the oscillation indices are both dependent upon material constants for the viscoelastic bi-materials interfacial crack. This is different from the corresponding elastic bi-materials problem. In the elastic bi-materials problem only the oscillation index is dependent on material constants, but the singularity index remains constant, i.e. $-1 / 2$, invariably. Apart from the mismatch of the elastic moduli of the materials on both sides of the interface, there is an additional viscosity mismatch for the viscoelastic bi-materials problem. It is the mismatch of viscosity that makes the singularity index deviate from $-1 / 2$ and dependent on material constants. It is also noted that the singularity index is related to mismatch of viscosity but independent of the amplitude of viscosity of the materials on both sides of the interface. The dependence of the singularity index and the oscillation index on the frequency of harmonic load is due to the fact that the complex moduli of viscoelastic materials are frequency-dependent. In addition, the viscosity of material is pronounced only within a finite range of frequency, i.e. the effective influence range of frequency, for the viscoelastic material which can be described by the standard lin- 
early solid model. Consequently, the dependence of the singularity and the oscillation index on the viscoelastic material constants is pronounced only within the effective influence range of frequency. Outside the effective influence range of frequency, the viscoelastic bi-materials problem does not distinguish from the elastic bi-materials problem.

In the case of non-harmonic load, the oscillating singular stress fields near the crack tip may be obtained theoretically in terms of a Fourier series, since a realistic load can be expanded in terms of a Fourier series. It is noted that each term in the series includes the singular factor $r^{\lambda(\omega)}$. On the other hand, due to the fact that the response of viscoelastic materials is dependent on the history of loading, the oscillating singular stress fields near crack tips are time-dependent and further, the singularity and the oscillation indices may be time-dependent, namely a singular factor $r^{\lambda(t)}$ may be included. The time-dependent oscillating singular stress fields may be obtained directly by solving the crack boundary-valued problem in the Laplace transformed domain and then performing inverse Laplace transformation to obtain the solution in the time domain instead of the Fourier series form in the frequency domain.

\section{References}

England, A.H. (1965). A crack between dissimilar media. Journal of Applied Mechanics 32, 400-402.

Erdogan, F. (1965). Stress distribution in bounded dissimilar materials with crack. Journal of Applied Mechanics 32, 403-410.

Erdogan, F. and Gupta, G.D. (1973). Numerical solution of singular integral equations. in: Mechanics of Fracture I. (edited by G.C. Sih), Noordhoff, International Publishing, Leiden, 368-425.

Freund, L.B. (1974). The stress intensity factor due to normal impact loading of the faces of a crack. International Journal of Engineering Science 12, 179-189.

Kuo, A.Y. (1984). Transient stress intensity factors of an interface crack between two dissimilar anisotropic halfspaces. Journal of Applied Mechanics 51, 71-76.

Lowengrub, M. and Sneddon, I.N. (1973). The stress field near a Griffith crack at the interface of two bounded dissimilar elastic half-planes. International Journal of Engineering Science 11, 1025-1034.

Muskhelishvili, I.N. (1953). Singular Integral Equations. Groningen: Noordhoff.

Qu, J.M. (1994). Interface crack loaded by a time-harmonic plane wave. International Journal of Solids Structure 31, 329-345.

Rice, J.R. and Sih, G.C. (1965). Plane problems of cracks in dissimilar media. Journal of Applied Mechanics 32, 418-423.

Srivastava, K.N., Gupta, O.P. and Palaiya, R.M. (1978). Interaction of elastic waves in two bounded dissimilar elastic half-spaces having Griffith crack at interface I. International Journal of Fracture 14, 145-154.

Williams, M.L. (1959). The stress around a fault or crack in dissimilar media. Bulletin of the Seismographic Society of America 49, 199-204. 